\title{
ESPÉCIES VEGETAIS TÓXICAS DA FAMÍLIA EUPHORBIACEA: UMA ANÁLISE SOBRE A DISTRIBUIÇÃO E OS CASOS DE INTOXICAÇÃO REGISTRADOS EM ARAGUAÍNA, TO
}

Toxic plant species of the Euphorbiaceae family: an analysis of the distribution and the cases of poisoning recorded in Araguaina, TO

Espécies vegetais tóxicas da família Euphorbiaceae: un análisis de la distribución y los casos de intoxicación registrado em Araguaína, TO

Jéssica Nayara Lopes Oliveira Lima ${ }^{1}$, Claudia Scareli-Santos ${ }^{*}{ }^{1}$, Suely Machado da Silva ${ }^{1}$, Helayne Carvalho Lima ${ }^{1}$, Jacqueline Silva Almeida ${ }^{1}$, Thainara Monteiro Parente ${ }^{1}$

${ }^{1}$ Curso de Licenciatura em Biologia, Universidade Federal do Tocantins, Araguaína, Tocantins, Brasil.

*Correspondência: Avenida Paraguai, s/n², esquina com a Rua Uxiramas Setor Cimba | 77824-838| Araguaína/TO.E-mail: scareliclaudia@uft.edu.br

\section{RESUMO}

O presente trabalho objetivou realizar um censo das espécies tóxicas da família Euphorbiaceae em Araguaína, TO, e avaliar quantos e quais foram os casos de intoxicação por plantas tóxicas. A pesquisa foi realizada em 35 bairros selecionados aleatoriamente. Destes bairros em 77,14\% foi encontrado Jatropha curcas e 22,86\% Euphorbia milii. Os três setores onde foram encontrados mais indivíduos de $J$. curcas foram Martins Jorge $(51,8 \%)$, São Miguel (55,5\%) e Céu Azul (70,37\%). Os valores da E. milii foram analisados em metros quadrados, a maior ocorrência foi registradas nos setores Manoel da Cunha $\left(2,05 \mathrm{~m}^{2}\right)$ e Palmas $\left(0,05 \mathrm{~m}^{2}\right)$. No período 2009 a 2015 foram notificados 37 casos de intoxicações, desses 30 são moradores de Araguaína, os outros são de cidades vizinhas; J. curcas foi responsável por $74 \%$ das intoxicações, os demais casos foram ocasionadas por Dieffenbachia picta (10\%) e em $36,66 \%$ não foi identificada a causa de intoxicação. A maioria dos casos registrados $(96,29 \%)$ está associada às crianças com faixa etária de um a dez anos. Os dados obtidos poderão ajudar na elaboração de material informativo sobre os casos de intoxicação, bem como quais espécies com maiores ocorrências, alertando a população em geral sobre os perigos da ingestão de plantas tóxicas.

Palavras-chave: Euphorbia milii; Jatropha curcas; Tocantins.

\section{ABSTRACT}

The present study aimed to conduct a census of toxic species in the family Euphorbiaceae present in Araguaína, TO and assess the number locations of poisoning cases by toxic plants. The survey was conducted in 35 randomly selected neighborhoods. Of these 35 sectors found in $77.14 \%$ Jatropha curcas and 22.86\% Euphorbia milii. The three sectors where more individuals of J. curcas, in Loteamento Jorge Martins were found (51.8\%), São Miguel (55.5\%) and Céu Azul (70.37\%). The values of E. milii were in $\mathrm{m}^{2}$, greater occurrence of this species in Manoel da Cunha (2.05 m2) and Palmas sector (0.05m2). In period 2009 -2015 were 37 cases of poisoning, 30 of these cases are city dwellers of Araguaína, these kinds J.curcas was responsible for $53.33 \%$ of poisoning, other cases was caused by Dieffenbachia picta (10\%), 36.66\% the cause of the poisoning species was not identified. The majority of the cases registered (96.29\%) is associated with children aged from one to 10 years old. The results may help in the preparation of informative material about the poisoning cases, as well as species related with the highest occurrence, alerting the population about the dangers of eating poisonous plants. 
Keywords: Euphorbia milii; Jatropha curcas; Tocantins.

\section{RESUMEN}

Este estudio tuvo como objetivo la realización de un censo de las especies tóxicas de la familia Euphorbiaceae en Araguaína, TO, y evaluar cuántos y cuáles fueron los casos de intoxicación por plantas tóxicas. La encuesta se realizó en 35 barrios seleccionados al azar. Estos barrios 77,14\% se encontró Jatropha curcas y en $22.86 \%$ Euphorbia milii. Los tres sectores en los que se encuentran más individuos de J. curcas fueron Jorge Martins (51,8\%), São Miguel $(55,5 \%)$ y Céu Azul (70,37\%). Los valores de E. milii se analizaron en metros cuadrados, la mayor incidencia se registró en los sectores Manoel da Cunha $\left(2,05 \mathrm{~m}^{2}\right)$ y Palmas $\left(0,05 \mathrm{~m}^{2}\right)$. En el período 20092015 se notificaron 37 casos, donde 30 son residentes de Araguaína, otros son de los pueblos vecinos; J. curcas fue responsable en $74 \%$ de las intoxicaciones, otros casos fueron causados por Dieffenbachia picta (10\%) y el 36,66\% no identificó la causa de la intoxicación. La mayor parte de los casos reportados $(96,29 \%)$ se asocia con los niños de uno a diez años. Los resultados pueden ayudar en el desarrollo de materiales de información sobre casos de envenenamiento, y qué especies con gran ocurrencia, alertando a la población sobre los peligros del consumo de plantas tóxicas.

Descriptores: Euphorbia milii; Jatropha curcas; Tocantins.

\section{INTRODUÇÃO}

Nos dias atuais, mais que em qualquer outra época, os assuntos relacionados à Biologia despertam o interesse do público em geral e no que se referem às plantas tóxicas muitas vezes se observa pouco conhecimento sobre as mesmas. Estas costumam ser belas, apresentando flores vistosas, coloridas e atrativas, trazendo riscos às pessoas que as desconhecem e fazem uso inadequado das mesmas seja como ornamentais, comestíveis ou medicinais (MOURA e AGRA, 1989; BARG, 2004; FELIPPE e TOMASI, 2009; TEIXEIRA et al., 2013).

Segundo Oliveira et al. (2007) cerca de 70\% dos casos de intoxicação por plantas são acidentais e atingem pessoas entre 12 a 17 anos de idade. Os autores relatam que no caso dos adultos as ocorrências mais frequentes são as consideradas abusivas, ou seja, decorrentes do consumo voluntário de plantas por propriedades já conhecidas. Entre os sintomas do contato com as substâncias tóxicas vegetais, temos irritações na pele e mucosas, complicações gastrintestinais, cardíacas e neurológicas.
As espécies vegetais tóxicas, muitas vezes desconhecidas pela população, são utilizadas como ornamentais em casas, estabelecimentos comerciais, escolas e jardins públicos. Estas espécies devem ser desprovidas de princípios tóxicos ou elementos suscetíveis de provocar reações alérgicas nas pessoas (CAVALCANTI et al., 2003). Entre as substâncias tóxicas mais comuns presentes nos vegetais estão os alcaloides, os glicosídeos cardiotóxicos, os glicosídeos cianogenéticos e as toxoalbuminas (TAIZ e ZEIGER, 2009). Os alcaloides são substâncias naturais, nitrogenadas, de natureza básica, com acentuada ação farmacológica, embora apresentam papel importante na medicina, estes compostos são responsáveis por intoxicações em humanos (COSTA, 1972; FELIPPE e TOMASI, 2009).

As espécies tóxicas com alcaloides mais comuns são Atropa belladona L. e Datura suaveolens Humb \& Bonpl. ex. Willd, ambas pertencentes à família Solanaceae, Delphinium consolida L., da família Ranuculacea e Senecio braziliensis Less., da família Asteraceae. A presença de alcaloides beladonados em A. belladona e D. suaveolens já foi comprovada cientificamente e asseguram a essas 
espécies propriedades psicotrópicas. Esses alcaloides são agentes anticolinérgicos que inibem a ação da acetilcolina em efetores autônomos inervados pelos nervos pós-ganglionares colinérgicos, bem como na musculatura lisa, que é desprovida de inervação colinérgica (GOODMAN e GILMAN, 2006).

Os glicosídeos são substâncias frequentes no reino vegetal constituídos por uma fração de açúcar e outra conhecida como aglicona ou genina. Estas duas frações podem ser separadas por hidrólise enzimática ou por meio de ácidos (COSTA, 1972). As plantas com glicosídeos, embora muitas de interesse medicinal, são também responsáveis por intoxicações, como acontece com aquelas que contêm glicosídeos cardiotóxicos, presentes em Nerium oleander L. (Apocynaceae), Thevetia peruviana (Pers.) K. Schum. (Apocynaceae) e Asclepias curassavica L. (Asclepiadaceae) e cianogenéticos, como em Manihot esculenta Crantz (Euphorbiaceae). Toxoalbuminas são proteínas altamente tóxicas, quimicamente semelhantes a certas toxinas de bactérias, com acentuado efeito irritante gastrintestinal e ação hemoaglutinante (COSTA, 1972; FELIPPE e TOMASI, 2009). Entre as plantas com toxoalbuminas mais frequentes temos Jatropha curcas L., (Euphorbiaceae), considerado altamente tóxica devido à presença de ésteres de forbol que são responsáveis pelos efeitos nocivos que provocam nos seres humanos, como atividades carcinogênicas e ação inflamatória. Em seguida temos Ricinus communis L. (Euphorbiaceae) e Allamanda cathartica L. (Apocynaceae). Todavia substâncias como terpenos, terpenóides, compostos fenólicos, saponinas, cumarinas entre outras podem estar presentes atribuindo caráter tóxico à planta (COSTA, 1972; BARG, 2004; FELIPPE e TOMASI, 2009).
A família Euphorbiaceae é constituída por plantas de hábito bastante variado, desde ervas, subarbustos, árvores e lianas, com folhas inteiras ou compostas, e geralmente latescentes (JOLY, 1987). O gênero Euphorbia, que dá nome á família, é marcado pela presença de uma inflorescência de flores reduzidas, rodeadas por brácteas e glândulas. (OLIVEIRA et al., 2003).

Ainda segundo estes autores, existem três espécies vegetais pertencentes à família Euphorbiaceae responsáveis por grande número de intoxicações em crianças com idade entre três e 12 anos; estes dados foram obtidos em um levantamento realizado na cidade de Ribeirão Preto, SP. Os relatos desses autores apontam as espécies Euphorbia milii Des Moulins (coroa-de-Cristo), J. curcas (pinhão) e $R$. communis (mamona).

Os estudos direcionados às plantas tóxicas são de suma importância, pois, ainda é preocupante o número de pessoas que dão entrada em hospitais com intoxicações. Segundo O Sistema Nacional de Informações Tóxico-Farmacológicas (SINITOX) no ano de 2012 ocorreram 993 casos de intoxicação no país em 2012, sendo 629 (63,34\%) na faixa etária de zero a 14 anos (FUNDAÇÃO OSWALDO CRUZ, 2015). Segundo Oliveira et al. (2003) muitos os casos de intoxicação que não são notificados nos hospitais e nas unidades de pronto atendimento, provavelmente porque o intoxicado não procura atendimento médico ou ainda quando chega ao hospital não traz parte da planta responsável pela intoxicação ou, ainda, pela falta de conhecimento do profissional de saúde sobre a identificação da planta tóxica.

Ainda segundo estes autores, nos Estados Unidos da América os centros de controle de 
intoxicação foram procurados por mais ou menos 1,5 milhões de indivíduos por ano com suspeita de intoxicação por plantas. No Brasil os registros de intoxicação estão associados na maioria das vezes às espécies J. curcas e J. gossypiifolia (Euphorbiaceae), ambas denominadas vulgarmente de pinhão; também ocorrem registros de T. peruviana (Apocynaceae), popularmente conhecida por chapéu-de-Napoleão e Dieffenbachia seguinte (Araceae), denominada comigo-ninguém-pode, esta última é uma espécie proveniente da região Amazônica conhecida como a campeã de acidentes entre as crianças de um a sete anos.

Assim, os objetivos deste trabalho foram realizar um censo das espécies vegetais tóxicas da família Euphorbiaceae presentes na arborização urbana de Araguaína, TO e avaliar quantos e quais foram os casos de intoxicação por plantas tóxicas notificados no período 2009 a 2015 no município.

\section{MATERIAIS E MÉTODOS}

O trabalho foi realizado na cidade de Araguaína, TO (Fig. 1) localizada na região norte do Estado do Tocantins, coordenadas geográficas $07^{\circ} 11^{\prime} 28^{\prime}$ de latitude sul, 48 $12^{\prime} 26^{\prime}$ de longitude oeste, com uma área de 4.000,416 $\mathrm{km}^{2}$ e uma população humana estimada de 170.183 habitantes (IBGE, 2015).

Figura 1. A direita e ao alto esta a localização geográfica do município de Araguaína no estado do Tocantins; no mapa está a ilustrado em cores os bairros/setores onde a pesquisa foi realizada.

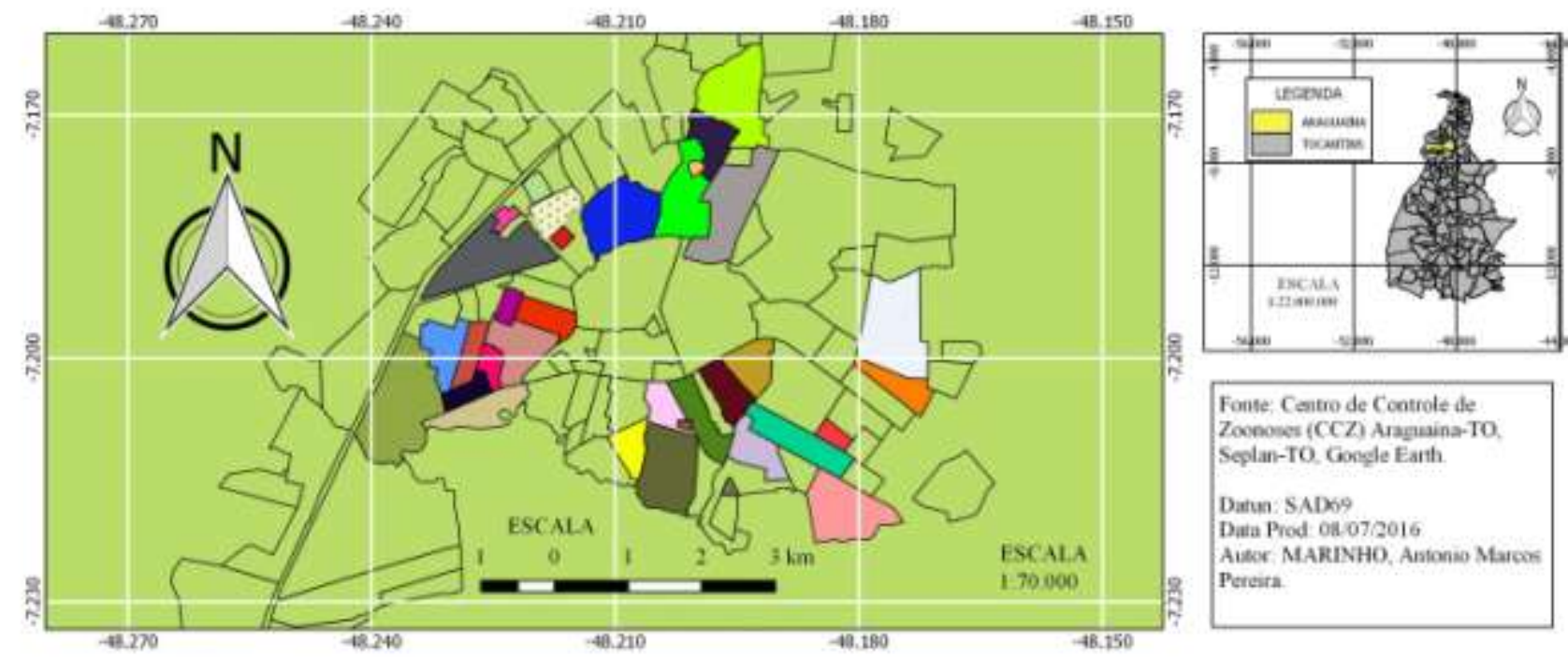

\begin{tabular}{|c|c|c|c|c|c|}
\hline \multicolumn{6}{|c|}{ LEGENDA } \\
\hline BAIRROS PARTKCIPANTES & Lee Manael Giones da Cuala & ] Jandian das Palmcires & Istoo Mingud & Sctir Neriestes & I Seter Vifa Nera \\
\hline$\square$ Sctar Sä Francesco. & Jar Maria & Inatim das Palmciras do Nortd & Setox Anhanguen & Ister Oante & Vila Goias \\
\hline Wvila da hes & Buire Eldorafo & I Jandim Esplanadu & |Sectur Ërasil & Sctur Palmas & IVib Nova \\
\hline EVia Farneina & $\square \mathrm{Cs}=\mathrm{Aral}$ & Jantim Iatiaia & Sctur Cruming & Seter Santa Leria & Vib Ribcivo \\
\hline Vila konario & Cimba & I Indim Palisea & | Sotur hapei & Iscear Sanus Tareainha & Jib Suntiages \\
\hline Teer Rodovaino & 15.2. Dom Crione & Marnata de Sol & I Selox Martian banpe & IStar tintases & \\
\hline
\end{tabular}


Para a análise dos casos de intoxicação por plantas, acidentais ou não, ocorridos e notificados nos hospitais e pronto atendimento do município de Araguaína, TO, no período de 2009 a 2015, foram examinadas as planilhas, disponibilizadas pelo Centro de Controle de Zoonoses (CCZ). Os dados receberam tratamento estatístico adequado para seguintes variáveis idade, sexo do paciente, tipo de intoxicação, nome científico e popular da planta ingerida (OLIVEIRA et al., 2003).

Foram realizados levantamentos e análises da arborização urbana enfocando espécies da família Euphorbiaceae, primeiramente foram listados todos os bairros e posteriormente escolhido aleatoriamente 35 bairros do município de Araguaína, que são os seguintes: Aeroporto, Ana Maria, Anhanguera, Bairro Eldorado, Jardim das Palmeiras, Jardim Esplanada, Jardim Itatiaia, Jardim Paulista, Morada Do Sol, Noroeste, Loteamento Cruzeiro, Loteamento Martins Jorge, Vila da Paz, Vila Ferreira, Vila Goiás, Vila Nova, Vila Ribeiro, Vila Rosário, Vila Santiago, Santa Luzia, Brasil, Céu Azul, Cimba, Dom Orione II, Itaipu, Jardim Palmeira do Norte, Manoel da Cunha, Noroeste, Oeste, Palmas, Santa Luzia, Santa Terezinha, São Francisco, São Miguel e Urbano (Fig. 1). Os indivíduos das espécies de Euphorbia milii foram analisados em metros quadrados. Os resultados foram organizados em um banco de dados informatizado com as seguintes informações: nome comum e suas variações regionais, família botânica, nome científico, origem, porte e características morfológicas das espécies.

\section{RESULTADOS E DISCUSSÃO}

3.1 Censo das espécies tóxicas da família Euphorbiaceae presente nas ruas e avenidas da cidade de Araguaína, TO.

Os resultados obtidos no censo dos 35 bairros/setores amostrados no município demonstraram que $77,14 \%$ destas localidades apresentam indivíduos de Jatropha curcas e 22,86\% correspondeu a Euphorbia milii. Flores foram observadas em ambas as espécies e frutos somente em J. curcas.

A espécie $J$. curcas é originária da América tropical, das regiões áridas do Nordeste brasileiro, entretanto desenvolve-se bem em toda parte tropical de todo o globo terrestre (FELIPPE e TOMASI, 2009); possui crescimento rápido em solos pedregosos e de baixa umidade (MAKKAR et al., 1998; GANDHI et al., 1995). Apresenta diferentes nomes populares no Brasil dentre eles pinhão, pinhão manso, pinhão-de-purga, pinhão branco, pinhão paraguaio, figo-do-inferno; na Colômbia é denominado purga de fraile (MATOS et al., 2011). Não apresenta valor ornamental considerável, e está mais associado ao uso na medicina não tradicional como cicatrizante, purgativo, para tratamentos de inflamações, hematoma, sinusite e hidropisia (BERG, 2010). A propagação de suas sementes ocorre pelos ventos o que possibilita sua colonização em áreas urbanas bem como nas periféricas. Segundo Matos et al. (2011), esta espécie possui inflorescências cujas flores são pequenas, com coloração amarelo-esverdeadas e reunidas em cimeiras; com fruto típico no formato de cápsula com $2,5 \mathrm{~cm}$ de comprimento, são extremamente lisos, com consistência coriácea e apresentam internamente três lóculos com uma semente cada. 
As intoxicações são causadas pelos ésteres do forbol que causam ação irritante e inflamatória na pele, mucosas e olhos. Estes ésteres estão presentes em maior concentração no látex de $J$. curcas, que é exsudado da planta quando é manipulada, seja durante a atividade de jardineiros bem como por crianças durante as brincadeiras infantis; as sementes também são tóxicas quando são ingeridas in natura (FELIPPE e TOMASI, 2009; MATOS et al., 2011).

A maior ocorrência de indivíduos de $J$. curcas foi observada no setor Céu Azul (70,3\%), seguida pelo Loteamento Martins Jorge $(51,85 \%)$ e o Setor São Miguel (55,55\%). Os bairros menos representativos foram Aeroporto, Jardim Itatiaia, Loteamento Cruzeiro, Morada do Sol, Vila Ribeiro, Dom Orione II, Itaipu, Jardim Paulista e Jardim das Palmeiras com $3,70 \%$ cada (Tab. 1). Todos os indivíduos amostrados nos 35 bairros apresentaram flores e fruto.

Os sintomas da intoxicação reportadas em seres humanos são marcados inicialmente por diarreia seguida por irritação gastrointestinal caracterizada por náuseas, vômitos, dores abdominais fortes $\mathrm{e}$ evacuações sanguinolentas. Em casos graves ocorreram espasmos musculares, entorpecimento, complicações respiratórias, hipotensão e desidratação (MATOS et al., 2011).

Tabela 1. Distribuição do número de indivíduos de Jatropha curcas L. amostrados nas vias públicas do município de Araguaína, TO, e agrupados em intervalos de altura em centímetros.

\begin{tabular}{cc} 
Altura em cm & Número de indivíduos \\
\hline$>$ que $40 \mathrm{~cm}$ & 22 \\
$40-80$ & 22
\end{tabular}

\begin{tabular}{cc}
$81-120$ & 27 \\
$121-160$ & 28 \\
$161-200$ & 24 \\
$201-240$ & 4 \\
$<250 \mathrm{~cm}$ & 1 \\
\hline
\end{tabular}

Entretanto J. curcas tem sido utilizado na medicina caseira, o óleo extraído das sementes tem emprego no tratamento da gota e das doenças reumáticas e o macerado das folhas é usado para o controle de hemorragias (FELIPPE e TOMASI, 2009); o chá das folhas de J. curcas é usado popularmente para curar dores de cabeça, constipação nasal, febre e fraqueza, apesar de todas as partes do vegetal apresentarem, em maior ou menor grau, propriedade nocivas para o homem e os animais (MATOS et al., 2011). Provavelmente essa pode ser também umas das causas de intoxicação por esta espécie, evidenciando a falta de informação voltada para a população leiga.

Os indivíduos da espécie de $J$. curcas apresenta hábito arbustivo, que pode chegar de 3-4 m de altura, latescente e alta resistente á seca (MATOS et al. 2011). Já Euphorbia milii é uma arbusto perene, latescente, com cerca de um metro e meio de altura, apresentando ramos compridos, contorcidos e em grande número, portando espinhos com comprimento médio de $2,5 \mathrm{~cm}$; apresentam diferentes nomes populares, como coroa-de-cristo, dois irmãos e coroade-Nossa-Senhora (OLIVEIRA et al., 2003).

Indivíduos de E. milii foram amostrados somente em 22,88\% dos bairros participantes da amostragem (Tab. 2). Foi observada maior ocorrência desta espécie no setor Manoel da Cunha $\left(2,05 \mathrm{~m}^{2}\right)$ e no setor Palmas $\left(0,05 \mathrm{~m}^{2}\right)$. Esta espécie apresenta valor ornamental, é utilizada muitas vezes em canteiros 
como "cerca-viva" junto aos muros das residências, pois além de adornar possibilita certa segurança devido à presença de espinho e acredita-se que dessa forma consiste em uma barreira de entrada de intrusos.

Segundo Oliveira et al. (2003), os casos mais frequentes de intoxicação por E. milli estão associados ao contato do látex com a pele e com as mucosas, o qual promove queimaduras que podem ser agravadas na presença de sol. Também já foram relatados incidentes em adultos devido à atividade de jardinagem e poda, onde o látex pode causar irritação na pele e nos olhos, seguida de conjuntivite e, caso não seja tomado cuidados médicos, pode ocasionar cegueira.

Tabela 2. Ocorrência em metros quadrados dos canteiros da espécie E. milii no município de Araguaína, TO.

\begin{tabular}{cc}
\hline Bairros/Setores & Metros quadrados \\
\hline Manoel da Cunha & 2,05 \\
São Francisco & 1,78 \\
Noroeste & 1,02 \\
São Pedro & 0,75 \\
São Miguel & 0,6 \\
Jardim das Palmeiras & 0,44 \\
Jardim Paulista & 0,42 \\
Eldorado & 0,26 \\
Céu Azul & 0,20 \\
Brasil & 0,14 \\
Santa Luzia & 0,07 \\
Palmas & 0,05 \\
\hline
\end{tabular}

Em crianças, o contato ocorre frequentemente durante as brincadeiras, ao tirar "leite da planta para fazer comidinha". Casos de ingestão são mais raros, provavelmente devido ao sabor desagradável do vegetal e ao rápido aparecimento de irritações na pele e mucosa oral.

\subsection{Casos de intoxicação notificados junto à Secretaria Municipal de Saúde de Araguaína, TO.}

Durante o período de 2009 a 2015, foram notificados 30 casos de intoxicações em Araguaína, TO, sendo que a espécie $J$. curcas foi responsável por $53,33 \%$ das intoxicações, os demais casos de intoxicação foi ocasionada por Dieffenbachia picta $(10,00 \%)$, por espécies não identificadas $36,66 \%$.

As circunstâncias de intoxicação por parte da família Euphorbiaceae foi $100 \%$ acidental, acredita-se que a maioria do público leigo desconhece os princípios tóxicos dessas plantas. Entre as outras plantas foram relatados casos de automedicação, bebida, prática de aborto e tentativa de suicídio.

Foram notificados casos de intoxicação por $J$. curcas em 17 localidades. Os bairros do município de Araguaína que apresentaram maior número de casos de intoxicação foram a região central com $24,30 \%$, seguida pelo Araguaína Sul com 16,20\%. Os resultados evidenciaram também que as notificações ocorreram em bairros localizados tanto no centro da cidade como na periferia (Tab. 3).

Analisando a distribuição dos casos de intoxicação, notificados pela Secretaria Municipal de Saúde, por plantas da família Euphorbiaceae, detectamos 16 registros para a espécie J. curcas em 10 bairros, sendo a região central com maior número de ocorrências, totalizando $26,27 \%$ dos casos, seguida dos bairros Nova Araguaína e Centro com 12,13\% cada (Tab. 4).

Em relação ao sexo no total de 27 pessoas intoxicadas, $33 \%$ foram do sexo masculino, e $66 \%$ feminino. As intoxicações causadas por Jatropha curcas foi $31 \%$ masculino e $68,75 \%$ feminino. A 
grande maioria dos casos registrado de intoxicação foi com crianças, cerca 96,29\% com a faixa de um a dez anos, por serem bastante curiosas as crianças estão mais suscetíveis a intoxicação. As outras ocorrências foram na faixa de 12 até 58 anos.

Tabela 3. Percentual de ocorrências de intoxicações por espécies totais (identificadas e desconhecidas) em 17 bairros/setores de Araguaína, TO e notificados junto à Secretaria Municipal de Saúde.

\begin{tabular}{cc}
\hline Bairro/Setor & $\begin{array}{c}\text { Valores } \\
\text { percentuais }\end{array}$ \\
\hline Araguaína Sul & 16,20 \\
Centro & 24,30 \\
Jardim dos Ipês & 3,70 \\
Jardim Santa Helena & 3,70 \\
Nova Araguaína & 5,40 \\
Santa Luzia & 3,70 \\
Brasil & 5,40 \\
Maracanã & 5,40 \\
Palmas & 3,70 \\
Vila Goiás & 3,70 \\
Vila Norte & 3,70 \\
Vila Piauí & 3,70 \\
Raizal & 5,40 \\
Universitário & 3,70 \\
Monte Sinai & 8,10 \\
Dom Orione & 3,70 \\
Beira do Lago & 3,70 \\
\hline
\end{tabular}

Tabela 4. Percentual de ocorrências de intoxicações, notificadas junto à Secretaria Municipal de Saúde, por Jatropha curcas em 10 bairros de Araguaína, TO.

\begin{tabular}{cc}
\hline Bairro/Setor & $\begin{array}{l}\text { Valores } \\
\text { percentuais }\end{array}$ \\
\hline Araguaína Sul & 26,37 \\
Nova Araguaína & 13,33 \\
Centro & 13,33 \\
Jardim dos Ipês & 6,67 \\
Jardim Santa & 6,67 \\
Helena &
\end{tabular}

\begin{tabular}{cl} 
Setor Palmas & 6,67 \\
Setor Brasil & 6,67 \\
Setor Maracanã & 6,67 \\
Santa Luzia & 6,67 \\
Vila Goiás & 6,67 \\
\hline
\end{tabular}

Segundo Tavares et. al. (2013), as crianças possui características que as tornam mais vulneráveis aos acidentes, destacando-se a imaturidade física e mental, a inexperiência e incapacidade para prever e evitar situações de perigo, a grande curiosidade e motivação em realizar tarefas, tendência a imitar e repetir comportamentos, a desproporção crânio-corpo e as pequenas dimensões das vias aéreas superiores.

\section{CONCLUSÃO}

Com os resultados obtidos na pesquisa, concluímos que as espécies Jathorpha curcas L. e Euphorbia milii Des Moulin são as únicas representantes da família Euphorbiaceae presentes nas vias públicas da cidade de Araguaína, TO; e que os casos de intoxicação em humanos, notificados no município, estão associados principalmente às crianças e à uma única espécie representada por $J$. curcas com $53,33 \%$.

Finalizando, os dados obtidos neste trabalho evidenciaram a importância do tema pesquisado, entretanto surgiram preocupações como a disseminação do conhecimento obtido para a população em geral e também para os profissionais da área de saúde, que atuam no primeiro atendimento ao paciente, e ao alto percentual de intoxicações por plantas não identificadas obtido, no período 20092015. Os dados obtidos poderão ajudar na elaboração de material informativo sobre os casos de intoxicação 
no município, bem como quais espécies apresentam maior ocorrência, alertando a população em geral sobre os perigos da ingestão de plantas tóxicas, assim evitando muitas intoxicações.

\section{AGRADECIMENTO}

Agradecemos ao Sr. Eduardo Freitas dos Santos, Diretor de Vigilância e Saúde do Centro de Controle de Zoonose e ao Sr. Rubens Carlos Neves, secretário municipal de saúde de Araguaína pela disponibilização dos dados referentes aos casos de intoxicação.

Todas as autoras declararam não haver qualquer potencial conflito de interesses referente a este artigo.

\section{REFERÊNCIAS}

BARG, D. G. Plantas tóxicas. Instituto Brasileiro de Estudos Homeopáticos, Faculdade de Ciências da Saúde de São Paulo, São Paulo. Monografia, 23p. 2004.

BERG, M. E. van der. Plantas medicinais na Amazônia: contribuição ao seu conhecimento sistemático. 3a ed., 266 p., 2010.

COSTA, A. F. Farmacognosia v.1, II $2^{\mathrm{a} e d ., ~ F u n d a c ̧ a ̃ o ~}$ Calouste Gulkenkian, 1172 p., 1972.

FELIPPE, G. M.; TOMASI, M. C. Venenosas: Plantas que Matam Também Curam. Editora SENAC, 352 p. 2009.

FUNDAÇÃO OSWALDO CRUZ. Casos registros de Intoxicação Humana por Agente Tóxico e Trimestre. Brasil. Rio de Janeiro, 2012. Disponível em: <http://www.fiocruz.br/sinitox>. Acesso em: 14. mar. 2016.

GOODMAN, L.S.; GILMAN, A. As Bases Farmacológicas da Terapêutica. $11^{\mathrm{a}}$.ed., Mc GrawHill Internacional do Brasil, 1821p., 2006.
GANDHI, V.M.; CHERIAN, R, M.; MULKY, M, J. Toxicological studie on ratanjyout oil. Food Chemical Toxicology. v. 33, n. 1, p. 33-42, 1995.

IBGE, Instituto Brasileiro de Geografia e Estatística. Disponível em: <https://www.ibge.gov.br> Acesso em: 20 jul. 2013. JOLY, A, B. Botânica: Introdução a Taxonomia Vegetal. 5 $^{\text {a }}$ ed. Editora Nacional, 777p., 1987.

MAKKAR, H, P, S.; ADERIBIGDE, A, O.; BECKER, K. Comporative evalution of non-toxic and toxic varieties of Jatropha curcas for chemical composition, digestiblity, protein degradability and toxic factor. Food Chemistry. v.62, n.2, p. 207-215, 1998.

MATOS, F. A. J.; LORENZI, H. SANTOS, L. F. L.; MATOS, E. O.; SILVA, M. G. V.; SOUSA, M. P. Plantas Tóxicas: Estudo de fitotoxicologia química de Plantas Brasileiras. $1^{\text {a }}$ edição, Instituto Plantarum, 256 p., 2011.

MOURA, M. D. B.; AGRA, M. F. Apocynaceae tóxicas e medicinas ocorrentes nos estados de Pernambuco e Paraíba, Brasil. Acta Botanica Brasilica. v.3, n.2, p. 273-279. 1989.

OLIVEIRA, R.; GODOY, S, A, P.; COSTA, F, B. Plantas Tóxicas: Conhecimento e Prevenção de Acidentes. Holos, 64 p., 2003.

OLIVEIRA, R.; GODOY, S, A, P.; COSTA, F, B. Intoxicações com espécies da família Euphorbiaceae. Revista Brasileira de Biociências. v.5, n.1, p. 69-71, 2007.

TAIZ L.; ZEIGER, E. Fisiologia Vegetal. Artmed. 848 p., 2009.

TAVARES, E, O.; BURIOLA, A. A.; SANTOS J. A. T.; BALLANI, T. S. L.; OLIVEIRA, M. L. F. Fatores associados á intoxicação infantil. Escola Anna Nery v.17, n.1. p. 31-37, 2013.

TEIXEIRA, L. A. J.; MAZUTTI, A. R.; GONTIJO, E. E. L.; SILVA, M. G.; OGAWA, W. N. Aspectos toxicológicos de Thevetia peruviana e perfil dos usuários em Gurupi-TO. Revista Científica do ITPAC. v.6, n.4, p. 1-11, 2013 